07

\title{
Резонансное болометрическое детектирование широкополосных сигналов терагерцевого диапазона частот
}

\author{
(C) А.В. Богацкая ${ }^{1,2}$, Н.В. Кленов ${ }^{1,3,4, \text { I }, ~ П . М . ~ Н и к и ф о р о в а ~}{ }^{1,4}$, А.М. Попов ${ }^{1,2}$, А.Е. Щеголев ${ }^{3,4}$ \\ ${ }^{1}$ Московский государственный университет им. М.В. Ломоносова, физический факультет, Москва, Россия \\ ${ }^{2}$ Физический институт им. П.Н. Лебедева РАН, Москва, Россия \\ ${ }^{3}$ Московский технический университет связи и информатики, Москва, Россия \\ ${ }^{4}$ Научно-исследовательский институт ядерной физики им. Д.В. Скобельцына Московского государственного \\ университета им. М.В. Ломоносова, Москва, Россия \\ ฯE-mail: nvklenov@gmail.com
}

Поступило в Редакцию 29 апреля 2021 г.

В окончательной редакции 31 мая 2021г.

Принято к публикации 1 июня 2021 г.

\begin{abstract}
Обсуждается детектирование широкополосного излучения терагерцевого диапазона частот болометрическим способом с помощью гетероструктуры, состоящей из последовательности проводящих и диэлектрических слоев легированного и нелегированного полупроводника (арсенида галлия, германия). Такая структура формирует фотонный кристалл с разрешенными и запрещенными зонами (диапазонами поглощения и пропускания). Выбирая толщины проводящих и непроводящих слоев и уровни легирования, можно формировать спектральные интервалы эффективного поглощения, что позволяет детектировать импульсы в диапазоне частот $\geqslant 10^{12} \mathrm{~Hz}$ со спектральной шириной порядка несущей частоты.
\end{abstract}

Ключевые слова: детектор, болометр, терагерцевое излучение, резонанс.

DOI: 10.21883/PJTF.2021.17.51388.18850

Терагерцевое (THz) излучение все более активно проникает в различные области фундаментальной науки и техники [1-3]. Это, в частности, обусловливает растущий интерес к поиску эффективных широкополосных детекторов в THz-диапазоне. Наиболее перспективными здесь являются устройства на основе метаматериалов, представляющие собой различные двумерные конфигурации резонансных структур для достижения одно-, двух- или многополосного и широкополосного поглощения [4-6]. Однако большинство предложенных подходов сопряжено с разработкой сложных устройств на основе дорогостоящих высокотехнологичных методов и материалов. В настоящей работе предлагается использовать относительно простой резонансный болометрический метод детектирования электромагнитных сигналов, предложенный в $[7,8]$ и основанный на концепции оптико-механической аналогии явления квантово-механического туннелирования частиц через потенциальный барьер и проникновения электромагнитных волн через область со сверхкритической концентрацией электронов [9-11]. Также в работе анализируется возможность создания болометрического детектора с предельно большой спектральной шириной для детектирования THz-излучения.

Как отмечалось в [7], для болометрического метода детектирования электромагнитного сигнала принципиально важно его проникновение внутрь проводящей области, в которой происходит поглощение электромагнитной энергии, составляющее основу детекти- рования. Эффективность проникновения излучения в эту область можно существенно увеличить, если за проводящим слоем расположить диэлектрический слой, играющий роль электромагнитного резонатора: в таком случае в условиях совпадения частоты падающего излучения с частотой одной из собственных мод резонатора наблюдается туннельный резонанс прозрачности, приводящий к эффективному заполнению резонатора полем и, что важно, резкому увеличению поглощаемой в проводящем слое энергии. Такой детектор может быть использован для определения мощности квазимонохроматического излучения, ширина спектра которого не превышает ширины кривой резонансного поглощения. Увеличить „спектральную ширину детектора“ можно заменой одного проводящего слоя на гетероструктуру из периодической последовательности проводящих и диэлектрических слоев, выступающую в роли фотонного кристалла с зонной структурой спектра поглощения для распространяющегося в ней электромагнитного излучения. В таком случае ширина спектра детектируемого излучения определяется шириной разрешенной зоны фотонного кристалла. В [8] в качестве гетероструктуры была рассмотрена последовательность слоев кремния, разделенных слоями металла, расположенная на идеально отражающей поверхности. Существенной особенностью настоящей работы является исследование последовательности слоев нелегированного (толщина $a$ ) и легированного (толщина $d$ ) полупроводника, расположенного 
на идеально отражающей (металлической) поверхности. Диэлектрическую проницаемость такой структуры представим в следующем виде (необходимые данные для исследуемых в работе структур приведены в таблице):

$$
\varepsilon= \begin{cases}\varepsilon_{0} & \text { в диэлектрических слоях, } \\ \varepsilon_{0}-\frac{\omega_{p}^{2}}{\omega^{2}+v^{2}}+i \frac{\omega_{p}^{2} v}{\left(\omega^{2}+\nu^{2}\right) \omega} & \text { в проводящих слоях, } \\ \varepsilon_{\text {air }} & \text { в воздухе }\end{cases}
$$

где $\varepsilon_{0}$ - диэлектрическая проницаемость нелегированного слоя, в проводящих слоях мы использовали выражение из теории Друде [12], $\omega_{p}^{2}=4 \pi e^{2} n_{e} / m^{*}-$ плазменная частота, определяемая величиной $n_{e}\left(n_{e}-\right.$ концентрация носителей $n$-типа), $m^{*}$ - эффективная масса носителей $n$-типа, $v$ - эффективная (транспортная) частота столкновений, $\omega=2 \pi f-$ круговая частота излучения, $f-$ линейная частота. Например, при уровне легирования $n_{e}=10^{18} \mathrm{~cm}^{-3}$ значение плазменной частоты в германии $\omega_{p} \approx 7.6 \cdot 10^{13} \mathrm{~s}^{-1}$, при той же концентрации носителей в арсениде галлия плазменная частота примерно в 3 раза выше. Диэлектрическая проницаемость воздуха $\varepsilon_{a i r} \cong 1$. Использование легированного полупроводника вместо металла позволяет существенно менять концентрацию носителей в проводящих областях, а также более чем на порядок уменьшить транспортную частоту рассеяния носителей заряда в проводящих слоях. Как следствие, появляется возможность формировать зоны поглощения различной ширины и в различных частотных диапазонах, включая частоты порядка долей THz. Отметим, что среди наиболее распространенных в технике полупроводников, таких как кремний, германий, арсенид галлия, именно германий обладает наименьшей частотой столкновений носителей $n$-типа. Далее проводится сравнение результатов расчетов для германия и арсенида галлия.

Доля поглощаемой энергии, падающей на поверхность фотонного кристалла излучения, рассчитывалась нами по формуле [8]:

$$
\eta(\omega)=\frac{\omega_{p}^{2} v}{\omega^{2}+v^{2}} \frac{\int E^{2}(z) d z}{c E_{0}^{2}},
$$

где $E_{0}-$ амплитуда падающей волны, $E(z)$ получается из численного решения уравнения Гельмгольца, а интеграл в (2) берется по всем слоям легированного полупроводника. При этом возможными потерями энергии волны в диэлектрике мы пренебрегали.

Уравнение Гельмгольца решалось численно на сетке с таким пространственным шагом, что на размере проводящих слоев укладывалось не менее десяти узлов. При этом на границах проводящих и непроводящих слоев полагались непрерывными напряженность электрического поля и его производная $d E / d z$, а на отражающей поверхности, на которую нанесена структура $(z=0)$, мы полагали $E=0$.

Результаты расчетов величины $\eta(\omega)$ для разного числа слоев $N$ в структуре и различных значений толщины нелегированного слоя, определяющего положение зон поглощения, представлены на рис. 1. Все данные получены для заданного значения плазменной частоты в легированных слоях и, следовательно, различной степени легирования материалов. Именно в такой ситуации ширины зон оптического кристалла оказываются сопоставимыми. В случае простейшей структуры, соответствующей $N=1$, возникают узкие линии поглощения, положение которых определяется эффектом резонансного „туннелирования“ электромагнитного поля через область со сверхкритической концентрацией электронов при условии совпадения частоты поля с одной из собственных частот диэлектрического резонатора [7]:

$$
\Omega \approx \frac{\pi c}{a \sqrt{\varepsilon_{0}}} n, \quad n=1,2,3, \ldots
$$

При увеличении в структуре числа проводящих и непроводящих слоев каждый из резонансных пиков поглощения расщепляется на $N$ компонент, которые при достаточно больших $N$ сливаются в зону, аналогичную по своей природе разрешенной зоне в кристаллической решетке. Такие зоны в фотонном кристалле соответствуют эффективному поглощению энергии падающего излучения. Нетрудно видеть, что увеличение диэлектрической проницаемости непроводящего слоя позволяет минимизировать размер структуры для детектирования заданного диапазона частот.

Увеличение толщины нелегированных слоев ведет к формированию зон в области все более низких частот. В частности, оказывается возможным формирование зон поглощения в области частот $\omega \sim 1 \mathrm{THz}$ с шириной $\Delta \omega \sim 0.5-0.7 \mathrm{THz}$, что позволяет детектировать излучение в том числе в субтерагерцевом диапазоне частот с эффективностью на уровне 0.4. С другой стороны, увеличение числа слоев в структуре приводит к плавной зависимости коэффициента поглощения в пределах зоны, что позволяет эффективно детектировать сигналы с большой спектральной шириной. При этом из-за большей величины частоты столкновений носителей в GaAs по сравнению с таковой в $\mathrm{Ge}$ ширина линий поглощения в арсениде галлия оказывается больше, что приводит к более плавной зависимости $\eta(\omega)$ в пределах зоны в многослойной структуре.

Увеличения доли поглощаемой энергии и изменения ширины зон поглощения можно достичь, меняя степень легирования проводящих слоев структуры, обеспечивая при этом выполнение условия $\omega_{p}>\max \{\omega, v\}$. На первый взгляд кажется, что уменьшение концентрации электронов ведет к уменьшению поглощения 
Характеристики используемых в моделировании полупроводников

\begin{tabular}{c|c|c|c|c}
\hline $\begin{array}{c}\text { Полу- } \\
\text { проводник }\end{array}$ & $\begin{array}{c}\text { Диэлектрическая } \\
\text { проницаемость } \\
\varepsilon_{0}\end{array}$ & $\begin{array}{c}\text { Эффективная масса } \\
\text { носителей } n \text {-типа (в массах } \\
\text { свободного электрона) } m^{*}\end{array}$ & $\begin{array}{c}\text { Подвижность } \\
\text { носителей } n \text {-типа } \\
\mu_{m}, \mathrm{~cm}^{2} /(\mathrm{V} \cdot \mathrm{s})\end{array}$ & $\begin{array}{c}\text { Транспортная } \\
\text { частота } \\
\text { столкновений, } \\
\mathrm{s}^{-1}\end{array}$ \\
\hline $\mathrm{GaAs}$ & 10.9 & 0.067 & 8500 & $3.09 \cdot 10^{12}$ \\
$\mathrm{Ge}$ & 16.0 & 0.55 & 3900 & $8.2 \cdot 10^{11}$
\end{tabular}

Пр имечание. Транспортная частота вычислена по табличным значениям подвижности с использованием соотношения $\mu_{n}=e /\left(m^{*} v\right)$. Значения плазменной частоты определяются степенью легирования и при одной и той же степени легирования оказываются разными для исследуемых материалов, поскольку эффективные массы носителей $n$-типа существенно различаются.
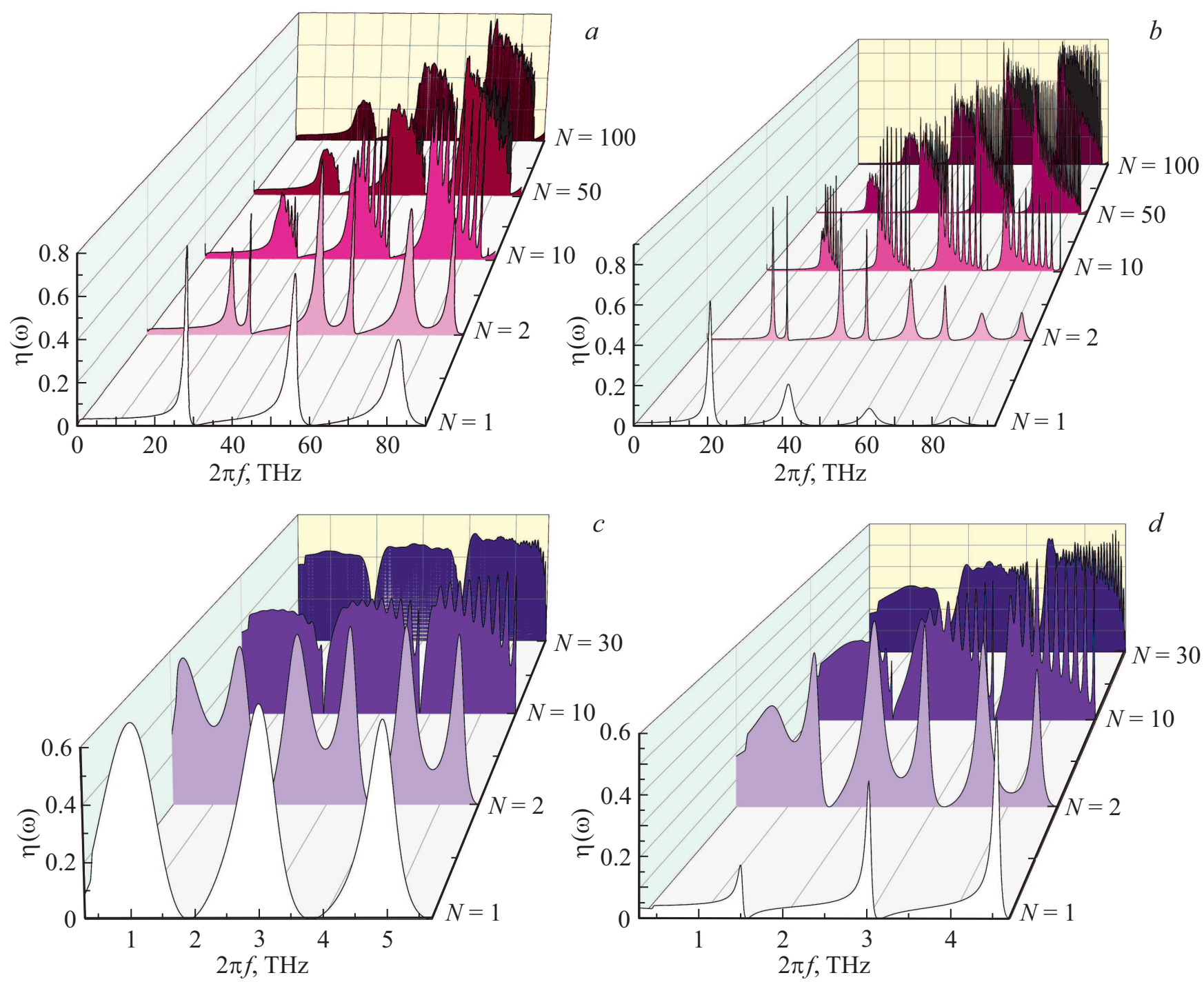

Рис. 1. Доля поглощаемой электромагнитной энергии, падающей на структуру из $\mathrm{GaAs}(a, c)$ и $\mathrm{Ge}(b, d)$, в зависимости от частоты излучения для различного числа используемых проводящих слоев $N . a, b-a=9 \mu \mathrm{m}, d=1 \mu \mathrm{m}, \omega_{p}=3 \cdot 10^{14} \mathrm{~s}^{-1}$ (уровни легирования: $\left.\mathrm{GaAs}-n_{e}=1.9 \cdot 10^{18} \mathrm{~cm}^{-3}, \mathrm{Ge}-n_{e}=1.56 \cdot 10^{19} \mathrm{~cm}^{-3}\right) ; c, d-a=150 \mu \mathrm{m}, d=0.5 \mu \mathrm{m}, \omega_{p}=6.9 \cdot 10^{13} \mathrm{~s}^{-1}($ уровни легирования: $\left.\mathrm{GaAs}-n_{e}=10^{17} \mathrm{~cm}^{-3}, \mathrm{Ge}-n_{e}=8.2 \cdot 10^{17} \mathrm{~cm}^{-3}\right)$.

излучения в структуре. Действительно, в соответствии с выражением (2) $\eta(\omega) \sim \omega_{p}^{2} \sim n_{e}$. С другой стороны, уменьшение концентрации электронов в легиро- ванных слоях приводит к более эффективной „туннельной связи“ непроводящих слоев, что сопровождается увеличением напряженности электрического по- 


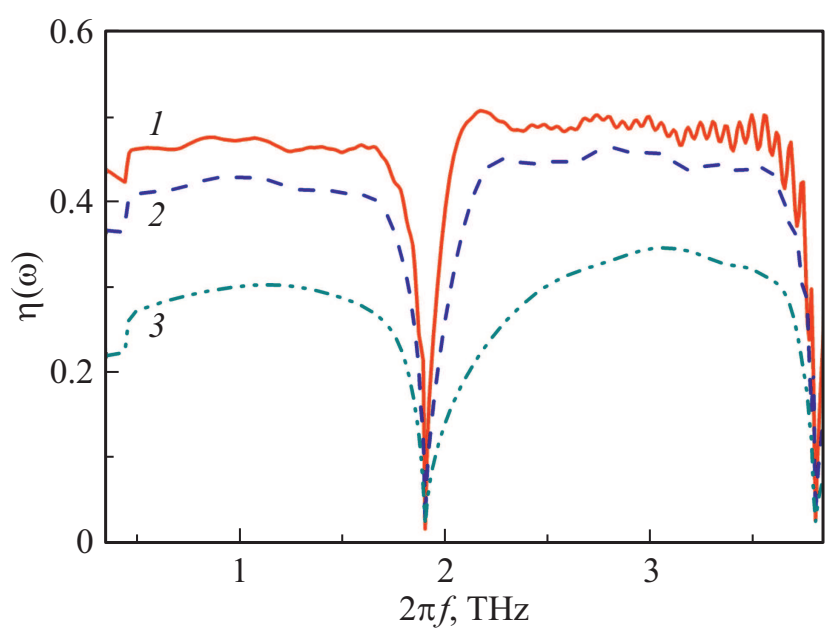

Рис. 2. Доля поглощаемой электромагнитной энергии, падающей на структуру из GaAs, в зависимости от степени легирования проводящих слоев. Концентрации носителей равны $5 \cdot 10^{16}(1), 10^{17}(2)$ и $3 \cdot 10^{17} \mathrm{~cm}^{-3}(3)$. Число слоев $N=30$. Параметры структуры: $a=150 \mu \mathrm{m}, d=0.5 \mu \mathrm{m}$.

ля в легированных областях (этот эффект описывается вторым сомножителем в выражении (2)). Расчеты (рис. 2) показывают, что в исследуемом диапазоне параметров с уменьшением концентрации носителей доля поглощенной энергии увеличивается, а спектральная ширина линии поглощения возрастает. Это обусловлено увеличением туннельной прозрачности проводящих слоев с уменьшением $n_{e}$. В результате можно достичь широкополосного поглощения THz-сигналов в области частот $\omega \sim 0.5-4 \mathrm{THz}$ с эффективностью на уровне $50 \%$ с узким провалом, соответствующим запрещенной зоне фотонного кристалла. Такая полоса частот, в частности, может быть удобна для детектирования импульсов (суб)пикосекундной длительности, а также последовательности импульсов, несущая частота которых меняется во времени от импульса к импульсу.

Как уже отмечалось, мы пренебрегали потерями энергии в непроводящих слоях гетероструктуры. Последние экспериментальные данные для арсенида галлия [13] в области частот $\sim 10^{12} \mathrm{~Hz}$ дают значение для тангенса угла потерь $\sim 3 \cdot 10^{-4}$. В такой ситуации действительно поглощение энергии в непроводящих слоях несущественно даже в структурах, содержащих более 100 слоев.

Проведенные расчеты показывают, что резонансное болометрическое детектирование может быть эффективно использовано для детектирования терагерцевых и субтерагерцевых импульсов излучения, в том числе с предельно большой спектральной шириной, с помощью гетероструктур, построенных на основе последовательности нескольких десятков легированных и нелегированных слоев таких материалов, как GaAs или Ge. При этом параметры структуры (толщины легирован- ных и нелегированных слоев, а также уровень легирования) должны подбираться в зависимости от конкретной полосы частот и длительности детектируемого сигнала.

\section{Финансирование работы}

А.В. Богацкая и П.М. Никифорова благодарят за поддержку грант Президента РФ МК-1932.2020.2. Анализ взаимодействия электромагнитных волн с тонкими слоями легированного полупроводника выполнен при поддержке Российского научного фонда (грант 20-69-47013). Н.В. Кленов - победитель грантового конкурса Стипендиальной программы Владимира Потанина 2020/2021.

\section{Конфликт интересов}

Авторы заявляют, что у них нет конфликта интересов.

\section{Список литературы}

[1] M. Hangyo, M. Tani, T. Nagashima, Int. J. Infrared Millim. Waves, 26 (12), 1661 (2005). https://doi.org/10.1007/s10762-005-0288-1

[2] X. Chai, X. Ropagnol, S.M. Raeis-Zadeh, M. Reid, S. Safavi-Naeini, T. Ozaki, Phys. Rev. Lett., 121 (14), 143901 (2018). https://doi.org/10.1103/PhysRevLett.121.143901

[3] H. Guerboukha, K. Nallappan, M. Skorobogatiy, Adv. Opt. Photon., 10 (4), 843 (2018). https://doi.org/10.1364/AOP.10.000843

[4] H. Tao, N.I. Landy, C.M. Bingham, X. Zhang, R.D. Averitt, W.J. Padilla, Opt. Express, 16 (10), 7181 (2008). https://doi.org/10.1364/OE.16.007181

[5] C. Gong, M. Zhan, J. Yang, Z. Wang, H. Liu, Y. Zhao, W. Liu, Sci. Rep., 6, 32466 (2016). https://doi.org/10.1038/srep32466

[6] W. Pan, T. Shen, Y. Ma, Z. Zhang, H. Yang, X. Wang, X. Zhang, Y. Li, L. Yang, Appl. Opt., 60 (8), 2235 (2021). https://doi.org/10.1364/AO.415461

[7] А.В. Богацкая, Н.В. Кленов, А.М. Попов, М.В. Терешонок, Письма в ЖТФ, 44 (15), 29 (2018). DOI: $10.21883 /$ PJTF.2018.15.46437.17354 [Пер. версия: 10.1134/S1063785018080059].

[8] А.Е. Щеголев, А.М. Попов, А.В. Богацкая, П.М. Никифорова, М.В. Терешонок, Н.В. Кленов, Письма в ЖЭТФ, 111 (7), 443 (2020). DOI: $10.31857 /$ S0370274X20070036 [Пер. версия: 10.1134/S0021364020070103].

[9] А.Б. Шварцбург, УФН, 177 (1), 43 (2007). DOI: $10.3367 /$ UFNr.0177.200701b.0043

[Пер. версия: 10.1070/PU2007v050n01ABEH006148].

[10] A.V. Bogatskaya, N.V. Klenov, M.V. Tereshonok, S.S. Adjemov, A.M. Popov, J. Phys. D, 51 (18), 185602 (2018). http://dx.doi.org/10.1088/1361-6463/aab756 
[11] A.V. Bogatskaya, E.A. Volkova, N.V. Klenov, M.V. Tereshonok, A.M. Popov, IEEE Trans. Antennas Propag., 68 (6), 4831 (2020).

DOI: 10.1109/TAP.2020.2972649

[12] Л.Д. Ландау, Е.М. Лифшиц, Электродинамика сплошных сред, 2-е изд., испр. (Наука, М., 1982), гл. IX.

[13] К.В. Маремьянин, В.В. Паршин, Е.А. Серов, В.В. Румянцев, К.Е. Кудрявцев, А.А. Дубинов, А.П. Фокин, С.С. Морозов, В.Я. Алешкин, М.Ю. Глявин, Г.Г. Денисов, С.В. Морозов, ФТП, 54 (9), 878 (2020). DOI: $10.21883 /$ FTP.2020.09.49825.17

[Пер. версия: 10.1134/S1063782620090195]. 